\title{
COMMUNICATION
}

Cite this: RSC Adv., 2013, 3, 22896

Received 14th August 2013

Accepted 3rd October 2013

DOI: $10.1039 / \mathrm{c} 3 \mathrm{ra} 44387 f$

\section{Versatile hydrogels: an efficient way to clean paper artworks $\uparrow$}

\author{
Claudia Mazzuca, ${ }^{a}$ Gianfranco Bocchinfuso, ${ }^{a}$ Ilaria Cacciotti, ${ }^{\text {ab }}$ Laura Micheli, ${ }^{a}$ \\ Giuseppe Palleschi ${ }^{a}$ and Antonio Palleschi ${ }^{\star a}$
}

www.rsc.org/advances

In this work we present innovative materials able to remove in a single, simple and not invasive treatment, different contaminants like starch paste from paper artworks. The materials, based on biocompatible hydrogels, overcome many of the problems usually faced by restorers during the cleaning of paper samples.

Paper artworks are difficult to restore, due to their inherent fragility, their degradation processes and their multi-component composition. A critical step during the restoration of these materials is the cleaning of the sheets (i.e., the removal of the dull patina). ${ }^{1}$ Moreover, past maintenance operations can complicate the scenario, as often the ancient paper artworks are strengthened by gluing external materials (i.e. other paper, wooden or cardboard lining paper) to the original piece (generally by using starch paste). This step often adds further problems, because the used glue undergoes to structural transformations that cause a loss in compactness, yellowing and an acidity increase, finally accelerating the degradation processes of the artwork itself. On the other hand, the cleaning techniques usually adopted are based on washing with solvents (organic or aqueous), and problems such as swelling and dissolution of some components during the treatments, often, cause subsequent problems. In addition, multi-steps treatments are often required to complete the cleaning procedure, being the properties of contaminants very different from a

"Dipartimento di Scienze e Tecnologie Chimiche, Università di Roma "Tor Vergata", Via della Ricerca Scientifica snc, 00133, Rome, Italy. E-mail: antonio.palleschi@ uniroma2.it; Fax: +3906 72594328; Tel: +3906 72594466

${ }^{b}$ Dipartimento di Ingegneria Industriale, Università di Roma "Tor Vergata", Via del Politecnico 1, 0013, Rome, Italy

$\dagger$ Electronic supplementary information (ESI) available: Information on materials, methods and adopted procedures. Graph reporting the change of the viscosities of the PLU and PEO hydrogels, as a function of shear rate, graph reporting the FTIR-ATR spectra for paper non treated or treated with PEO and PLU hydrogels and graph reporting the chromatograms of the hydrogels and of filter paper samples treated or not with PEO or PLU hydrogels. Table reporting the diffusional data obtained from the FRAP experiments for dextran 10000 and $70000 \mathrm{Da}$ and labeled amylase enzyme. See DOI: 10.1039/c3ra44387f chemical-physical point of view (i.e. hydrophilic, hydrophobic or, as for the glue, polymeric compounds). In the attempt to overcome a part of these problems, in the last years innovative cleaning methodologies have been proposed based on application of suitable gels. Due to the high retention power and viscosity of gels, the penetration of the liquids into the paper sheets is significantly reduced, therefore minimizing damages. ${ }^{2,3}$ However, to avoid dangerous microbial growth, ${ }^{4}$ a complete removal of the gel is required and such a procedure often needs abrasive mechanical action or solvents, often unsafe for the artwork. In this contest, reversible hydrogels ${ }^{5}$ represent a useful alternative to overcome many known problems. Nevertheless, unfortunately, to remove the glue components additional specific procedures are required; in this contest, for example the use of specific enzymes has been already proposed. ${ }^{3,6}$

Our basic idea has been to develop multi-component materials that collect the positive experiences reported in the literature to circumvent many of the problems faced by restorer during the cleaning of paper artwork. Furthermore, our ideal materials would have to be suitable to be used on samples presenting heterogeneous problems, as a consequence they had to be selectively usable on the contaminated regions. This last feature is difficult to obtain with conventional bath treatment; on the contrary, the hydrogel could give this advantage. In the present paper we have focused our attention on a well-known family of hydrogels; in particular we have used polyethylene oxides (PEO) or Pluronic copolymer poly(ethylene oxide) $)_{20^{-}}$ poly(propylene oxide $)_{70}$-poly(ethylene oxide $)_{20}\left(\mathrm{PEO}_{20}-\mathrm{PPO}_{70^{-}}\right.$ $\mathrm{PEO}_{20}$, in the following PLU), and $\alpha$-cyclodextrin (in the following, $\alpha$-CD) whose gelation is promoted by physical crosslinks induced by supramolecular self-assembling. ${ }^{7-10}$ The hydrogels based on physical cross-link are able to transduce external stimuli (like pressure) into macroscopical changes of their rheological properties (i.e. swelling) ${ }^{\mathbf{1 1 - 1 3}}$ In particular, the hydrogels investigated have a thixotropic behavior, as shown by the incongruity of the measured rising and declining viscosity curves as a function of shear rate (ESI Fig. S1 $\dagger$ ). This property 
gives them a great advantage in the field of our interest, because they can be applied as hydrogel and then easily removed simply by reducing their viscosity by a soft mechanical action (i.e. the gentle use of a soft brush). This procedure is less invasive than traditional methods and limits the problems arising from the removal operations. These hydrogels possess other peculiarities that make them suitable in the field of paper restoration: (i) their efficiency is little sensitive to $\mathrm{pH}$ and do not require the presence of specific ions ${ }^{\mathbf{1 0}}$ allowing a fine tuning of the conditions adopted (i.e., presence of divalent, instead of monovalent, ions or use of alkaline $\mathrm{pH}^{\mathbf{1 4}}$ ); (ii) unlike other gels used for cleaning paper artworks, ${ }^{15}$ they are made up of synthetic molecules and therefore could be less susceptible to microbial attacks; (iii) their amphiphilic properties can be easily tuned, by slight changes in their composition, as described in more detail below; as a consequence they could be used to remove contaminants at different hydrophobicity degree. In this work, PEO and PLU hydrogels have been applied to paper samples, according to the procedures reported in ESI, $\dagger$ and then removed simply with a soft brush. The efficiency of the adopted removal conditions have been verified by means of visual inspections of the samples, by attenuated total reflectance-Fourier transform infrared (ATR-FTIR) analyses, and by HPLC analysis of the paper extracts after gel treatment and subsequent removal. In the ATR-FTIR experiments the absence of the polymers on the sample is confirmed by the inspection of the region 850$1200 \mathrm{~cm}^{-1}$ in the spectra, where the typical (different) bands of cellulose, PEO or PLU fall (ESI Fig. S2 $\dagger$ ). In all the cases, only the absorption bands of cellulose are present. The HPLC analysis confirms the absence of hydrogel components on the paper after the removal procedure, as the characteristic peaks of the used hydrogels are absent in the chromatogram of the extracts of treated samples (ESI Fig. S3†).

To test the efficiency of cleaning of the proposed hydrogel we have applied our hydrogel to three classes of samples (see $\mathrm{ESI}^{\dagger}$ for the adopted procedures):

1. Paper samples previously impregnated with linseed oil, as representative of fresh hydrophobic contamination.

2. Paper treated with linseed oil and then aged, as representative of old hydrophobic contamination. The obtained results have been compared with the well-established and traditional method that consists in an immersion in water. ${ }^{\mathbf{1 6}}$

3. Fragments of a real samples (from the volume "Theatrum Veritatis and Justitiae" - Venezia, 1735) have been treated with the studied hydrogel and the efficiency of acidic component removal has been tested. As for point 2, the samples have been cleaned also by immersion in water for comparison.

Concerning the point 1 , linseed oil has been chosen because it is a natural substance that can mimic oily contaminants and is widely used as a carrier for pigments in inks and as a primer for glossy paper. ${ }^{17}$ The FTIR-ATR spectra of the samples after and before treatment with PEO and PLU are reported in Fig. 1A. In this figure, it is possible to observe that after gel treatments, absorption peaks due to the carbonyl moiety in the oil (roughly centered at $\left.1740 \mathrm{~cm}^{-1}\right)^{18}$ in the spectra disappears, therefore indicating that oil is totally removed by using the hydrogels.

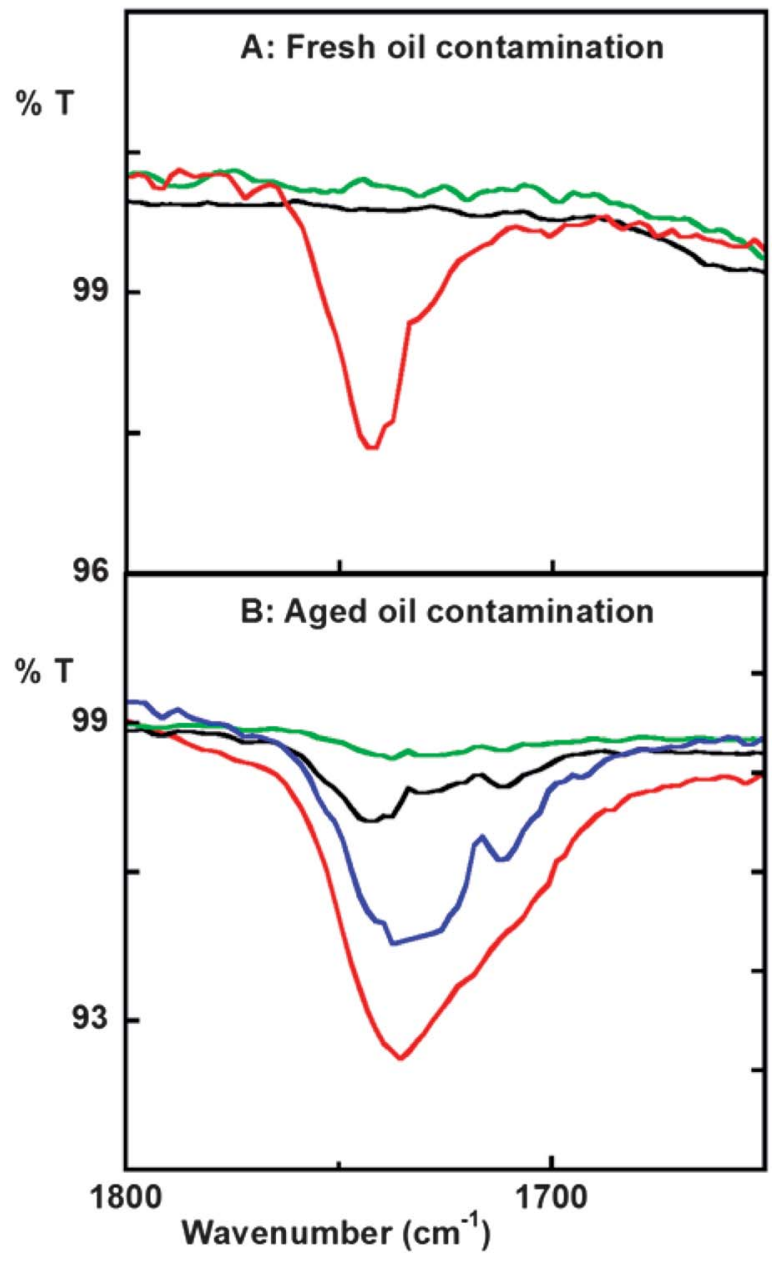

Fig. 1 FTIR-ATR spectra of paper samples. Paper after contamination (red line), samples contaminated and afterwards treated with PEO hydrogel (black line), PLU hydrogel (green line) and water (blue line). The graphs report data obtained on samples contaminated with fresh linseed oil (A) and aged linseed oil (B).

To reproduce the condition often faced in the restoration of ancient papers, we have artificially aged filter paper strips impregnated with linseed oil and then our hydrogels have been used to clean these "aged" samples (point 2). The efficiency of hydrogel in the cleaning of these strips has been compared to that of the commonly used method consisting in the washing of samples by immersion in water. Analysis by FTIR-ATR spectroscopy show that the two hydrogels have almost totally removed the oil, while the water treatment was not as much effective (Fig. 1B). Between PEO and PLU, the last is more effective than the former. This is coherent with the different nature of the two hydrogels; the presence of propylene oxide (in a $2: 1$ ratio respect to ethylene oxide) makes PLU slightly more hydrophobic than PEO (constituted only by ethylene oxide). ${ }^{19} \mathrm{It}$ is noteworthy than the procedure to obtain the two hydrogels is very simple and it is the same in the two cases; this gives to the restorers the opportunity to prepare "on-site" the materials that they need, changing the formulation as a function of the contaminant properties.

The aging of paper samples are often coupled with increasing of acidic components, that, in turn, promotes further 
sample degradations. For this reason, the removal of these components and the retrieval of safety $\mathrm{pH}$ values are crucial for the outcome of the maintenance and/or restoring operations. The efficiency of our materials in this contest has been tested by measuring the remaining fraction of organic acids after treatment of a real ancient sample (point 3), measured by means of HPLC analysis after extraction. The results are reported in Table 1. On the basis of comparison with organic acid standards, the peak at $7.5 \mathrm{~min}$ is assigned to malic acid, while that centered at $9.5 \mathrm{~min}$ is probably due to a degradation product of gelatine. The remarkable decrease of the peak area subsequent to the treatment, witnesses that the acidic components are removed by cleaning with hydrogels. By contrast, in the case of standard water treatment only a partial removal of malic acid is obtained. From this point of view, the hydrogel methods are more efficient than the traditional water bath. We have also measured the $\mathrm{pH}$ of the samples before and after treatment (Table 1). In all the cases the $\mathrm{pH}$ after treatments recovers the optimal value for cellulosic material ( $\mathrm{pH}$ about 9), with better results of PEO respect to PLU, suggesting that the former is more suitable in the treatment of hydrophilic contaminants.

Above we have shown the efficiency of the proposed hydrogels in the field of restoring/maintenance of the paper artworks. Another important issue in this contest is represented by the presence on the paper sheets of specific contaminations, difficult to remove by using the previous reported protocols. They are often represented by polymers, and, in general they require specific treatments, whose effect are difficult to predict. The most common example of this class of contamination is represented by the glues, often present because used in previous restoring actions. As an example of glue contaminants on the papers, we have used the starch. The starch glue, as known, can be digested by amylase enzyme., ${ }^{3,6}$ Between the other advantages of the hydrogels here presented, they are suitable to be used as carrier for enzymes, that can be added to confer to the formulated materials the ability to remove specific contaminants. In the last part of this communication we present the data obtained with composite materials constituted by $\alpha$-amylase enzyme trapped in PLU or PEO hydrogels. The preparation procedure remains very simple, and allows in situ encapsulation of the protein by using mild conditions at room temperature ${ }^{7}$ (see ESI $\uparrow$ for details). First of all we have verified the ability of polymers to diffuse in the investigated hydrogel, this is a preliminary test because diffusion properties are directly related with the availability of molecules on the hydrogel surface (in this case it is particularly important to

Table 1 Removal of acidic components from samples taken from the volume "Theatrum Veritatis and Justitiae" - Venezia, 1735

\begin{tabular}{|c|c|c|c|}
\hline \multirow[b]{2}{*}{ Sample } & \multicolumn{2}{|l|}{ HPLC } & \multirow[b]{2}{*}{$\mathrm{pH}$} \\
\hline & $\begin{array}{l}\text { Peak area } \\
\text { (a.u.) }\left(t_{\mathrm{R}} 7.5 \mathrm{~min}\right)\end{array}$ & $\begin{array}{l}\text { Peak area (a.u.) } \\
\left(t_{\mathrm{R}} 9.5 \mathrm{~min}\right)\end{array}$ & \\
\hline Before treatment & 12650 & 4287 & $7.8 \pm 0.1$ \\
\hline After water treatment & 2168 & 438 & $8.9 \pm 0.1$ \\
\hline After PEO treatment & 330 & $<10$ & $9.1 \pm 0.1$ \\
\hline After PLU treatment & 337 & $<10$ & $8.6 \pm 0.1$ \\
\hline
\end{tabular}

assure the presence of enzymes on the surface because very likely the glue hardly diffuses towards the enzyme in the hydrogel bulk, as is for smaller molecules). Fluorescence recovery after photobleaching (FRAP) experiments have been employed to evaluate the translational mobility of polymer molecules within the hydrogel (see $\mathrm{ESI}_{\dagger}^{\dagger}$ ). In particular, FITC-labeled dextrans with different molecular weight have been exploited as model compounds and the results compared with those obtained with FITC-labeled $\alpha$-amylase (ESI Table S1†). The decrease of the content of the mobile fraction in the case of 70000 Da dextran (respect to the $10000 \mathrm{Da}$ dextran), inferable from the decrease of the $k$ value in the table, suggests that the mesh size of the investigated hydrogel is roughly intermediate between the average dimension of the 10000 Da and 70 000 Da dextran (2.0 and 6.6 nm respectively), ${ }^{20,21}$ compatible with the diffusion of our enzyme (which hydrodynamic ratio, $R_{\mathrm{H}}$, is $3.2 \mathrm{~nm}$ ) The diffusion of $\alpha$-amylase in the hydrogels has been also tested. The lower value of diffusion coefficient in the hydrogel $\left(D_{\mathrm{E}}\right)$ obtained respect to that predictable on the basis of his $R_{\mathrm{H}}$ suggests that specific enzyme-hydrogel interactions take place. However, these interactions don't prevent the protein diffusion, ${ }^{\mathbf{2 1 , 2 2}}$ suggesting that the systems can be successfully used as cleaning agents.

As second step, the activity of the enzyme entrapped in the hydrogels has been checked on starch deposited on Petri dishes. The Fig. 2 reports the UV-Vis absorbance of the soluble starch-iodine complex spectra at different times of enzymatic PEO hydrogel treatment (see ESI $\dagger$ for details). These data show that the fraction of the solubilized starch, directly related with the enzymatic hydrolysis of the insoluble starch paste, increases with the hydrogel application time. The treatment with hydrogels without enzyme, on the contrary, is ineffective in the starch solubilization.

Starting from this promising evidences, the hydrogels have been used on real samples, and, as final test, the ability of the composite material to remove coated and aged starch paste from paper strips has been tested. To this end, we have applied the hydrogels containing $\alpha$-amylase enzyme for $45 \mathrm{~min}$, and then, after removal of the hydrogels, the paper strips were analyzed by FTIR-ATR spectroscopy (Fig. 3). As shown, in Fig. 3A, paper coated with starch paste, has a different FTIR-ATR absorption spectrum, respect to the paper alone. The

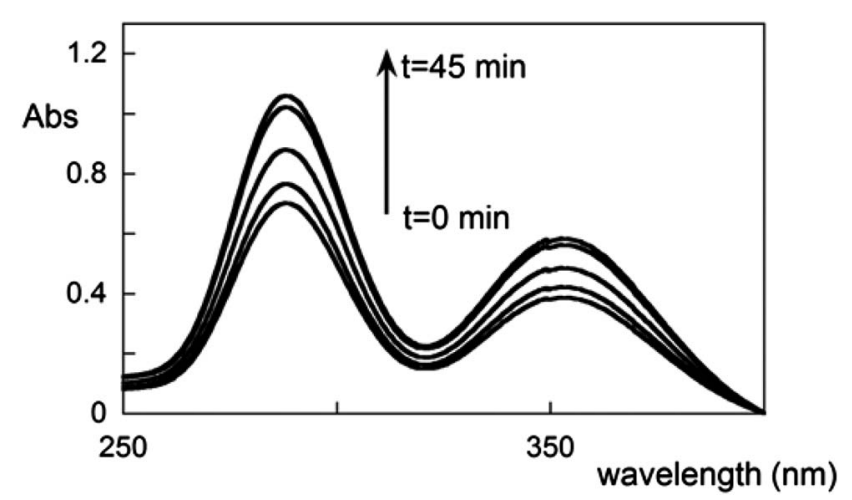

Fig. 2 UV-Vis absorbance spectra of the soluble starch-iodine complex as a function of PEO hydrogel application time. 


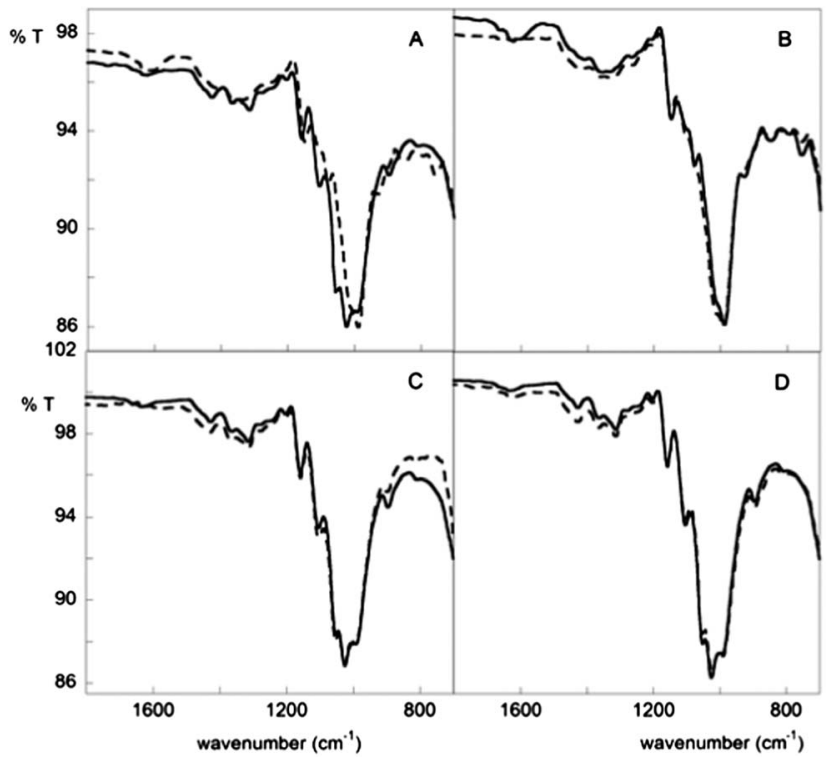

Fig. 3 Comparison of FTIR-ATR spectra of (A) paper (continuous line) and paper coated with starch paste (dashed line); (B) paper coated with starch paste cleaned using water bath (dashed line) or not (continuous line); (C) paper coated with starch paste cleaned with PLU hydrogel (dashed line) or PEO hydrogel (continuous line); (D) paper (dashed line) and paper coated with starch paste and cleaned with PEO hydrogel (dashed line).

characteristic peak of cellulose, centered at $1024 \mathrm{~cm}^{-1}$, is covered by the starch one, at about $998 \mathrm{~cm}^{-1} \cdot{ }^{23}$ These samples of paper coated with starch paste have been treated with traditional water washing, PEO or PLU loaded with $\alpha$-amylase. As shown in Fig. 3B, the water treatment does not restore the original cellulose spectrum, indicating that it is not able to remove the starch layer from the paper. On the contrary, as shown in Fig. 3C and D, the spectra of paper treated with hydrogel (PEO and PLU), are very similar to that of the paper alone, suggesting that treatment with the hydrogel is extremely effective in removing starch paste.

Overall, the results here reported represent the first example of an oncoming generation of new materials targeted to the cleaning of paper artworks; the $\alpha$-CD/polymer hydrogels are versatile, easy to prepare and functionalize, relatively cheap and safety. In this contest, the proposed works constitute the first and fundamental step of deeper studies, nonetheless, the presented materials show a great efficiency in all the investigated conditions, if compared with traditional methods. In particular, the ability to formulate on-site PEO or PLU hydrogels with the same protocol makes possible to treat samples with patina of different hydrophobicity. Furthermore, the possibility to easily trap $\alpha$-amylase can confer to these materials the possibility to remove efficiently starch glue.

\section{Acknowledgements}

This work was supported by the 'Progetto SIDE' of the Regione Lazio. The authors thank Prof. T. Coviello for helpful discussions and Drs R. Lettieri and E. Cervelli for technical support.

\section{Notes and references}

1 M. Strlič, J. Kolar and S. Scholten, Ageing and stabilization of paper, ed. M. Strlič and J. Kolar, Ljubljana National and University Library, Ljubljana, 2005, p. 1.

2 E. Carretti, I. Natali, C. Matarrese, P. Bracco, R. G. Weiss, P. Baglioni, A. Salvini and L. Dei, J. Cult. Herit., 2010, 11, 373.

3 A. Bluher, G. Banik and E. Thobois, International Conference of Conservation and Restoration of Archival and Library Materials: 22-29 April 1996, ed. C. Federici, P. F. Munafò and G. B. Palumbo, Palermo, 1999, p. 791.

4 D. Stulik, D. Miller, H. Khanjian, N. Khandekar, R. Wolbers, J. Carlson and W. C. Petersen, Research in Conservation Series 4, ed. V. Dorge, The Getty Conservation Institute, Los Angeles, 2004.

5 E. Carretti, M. Bonini, L. Dei, H. B. Berrie, L. V. Angelova, P. Baglioni and R. G. Weiss, Acc. Chem. Res., 2010, 43, 751.

6 I. Schwarz, A. Blüher, G. Banik, E. Thobois and K.-H. Maurer, Restaurator, 1999, 20, 225.

7 J. Li and X. J. Loh, Adv. Drug Delivery Rev., 2008, 60, 1000.

8 X. Ni, A. Cheng and J. Li, J. Biomed. Mater. Res., Part A, 2009, 88, 1031.

9 J. Li, A. Harada and M. Kamachi, Polym. J., 1994, 26, 1019.

10 J. Li, X. Li, Z. Zhou, X. Ni and K. W. Leong, Macromolecules, 2001, 34, 7236.

11 A. Palleschi, T. Coviello, G. Bocchinfuso and F. Alhaique, Int. J. Pharm., 2006, 322, 13.

12 T. Coviello, F. Alhaique, C. Parisi, P. Matricardi, G. Bocchinfuso and M. Grassi, J. Controlled Release, 2005, 102, 643.

13 W. L. Wu, M. Shibayama, S. Roy, K. Kurokawa, L. D. Coyne, S. Nomura and R. S. Stein, Macromolecules, 1990, 23, 2245.

14 M. Strlič, J. Kolar, D. Kočar and J. Rychly, Ageing and stabilization of paper, ed. M. Strlič and J. Kolar, Ljubljana National and University Library, Ljubljana, 2005, p. 111.

15 M. Bicchieri, M. Monti, G. Piantanida, F. Pinzari, S. Iannuccelli, S. Sotgiu and L. Tireni, Anal. Bioanal. Chem., 2012, 402, 1517.

16 S. Zervos and A. Moropoulou, Restaurator, 2003, 24, 160.

17 (a) F. Fiorani and G. Pace, Mitteilungen des Kunsthistorisches Institut in Florenz, 2008, 2, 240; (b) A. Gambaro, R. Ganzerla, M. Fantin, E. Cappelletto, R. Piazza and W. R. L. Cairns, Microchem. J., 2009, 91, 202.

18 (a) M. Lazzari and O. Chiantore, Polym. Degrad. Stab., 1999, 65, 303; (b) J. Mallégol, J.-L. Gardette and J. Lemaire, J. Am. Oil Chem. Soc., 2000, 77, 25; (c) M. D. Guillén, A. Ruiz, N. Cabo, R. Chirinos and G. Pascual, J. Am. Oil Chem. Soc., 2003, 80, 754 .

19 A. Sundblom, A. E. C. Palmqvist and K. Holmberg, Langmuir, 2010, 26, 1983.

20 F. Brandl, F. Kastner, R. M. Gschwind, T. Blunk, J. Teßmar and A. J. Göpferich, J. Controlled Release, 2010, 142, 221.

21 P. A. Perry, M. A. Fitzgerald and R. G. Gilbert, Biomacromolecules, 2006, 7, 521.

22 D. Venturoli and B. Rippe, Am. J. Physiol. Renal Physiol., 2005, 288, F605.

23 V. Librando, Z. Minniti and S. Lorusso, Conservation Science in Cultural Heritage, 2011, 11, 249. 